\title{
Broad Chiroptical Activity from Ultraviolet to Short-Wave Infrared by Chirality Transfer from Molecular to Micrometer Scale
}

Ki Hyun Park ${ }^{1}$, Junyoung Kwon ${ }^{1}$, Uichang Jeong ${ }^{1}$, Ji-Young Kim², Nicholas A. Kotov ${ }^{2,3,4}$, Jihyeon Yeom ${ }^{1,5,6,7 *}$

1 Department of Materials Science and Engineering, Korea Advanced Institute of Science and Technology (KAIST), 291 Daehak-ro, Yuseong-gu, Daejeon 34141, Republic of Korea

2 Department of Materials Science and Engineering, University of Michigan, Ann Arbor, MI 48109, United States

3 Department of Chemical Engineering, University of Michigan, Ann Arbor, MI 48109, United States

4 Biointerfaces Institute, University of Michigan, Ann Arbor, MI 48109, United States

5 Department of Biological Sciences, Korea Advanced Institute of Science and Technology (KAIST), 291 Daehak-ro,

Yuseong-gu, Daejeon 34141, Republic of Korea

6 Institute for Health Science and Technology, Korea Advanced Institute of Science and Technology (KAIST), 291 Daehak-

ro, Yuseong-gu, Daejeon 34141, Republic of Korea

7 Institute for the NanoCentury, Korea Advanced Institute of Science and Technology (KAIST), 291 Daehak-ro, Yuseong-gu,

Daejeon 34141, Republic of Korea 

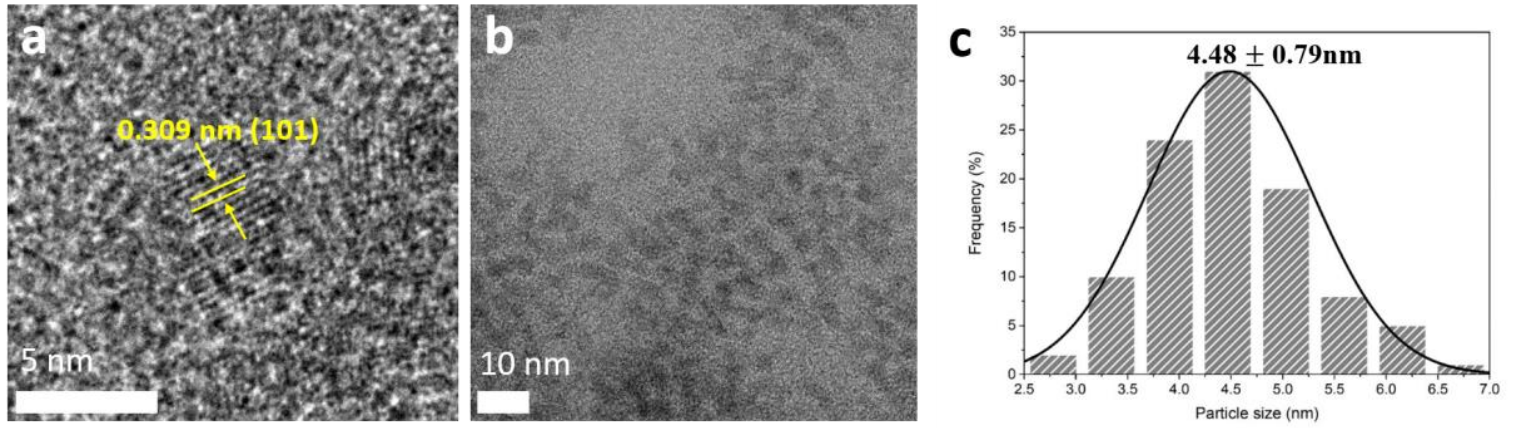

Figure S1. a) HRTEM, b) representative TEM image, and c) size distribution of synthesized $L$-Cys-Cu $\mathrm{Cu}_{2} \mathrm{NPs}$.

a

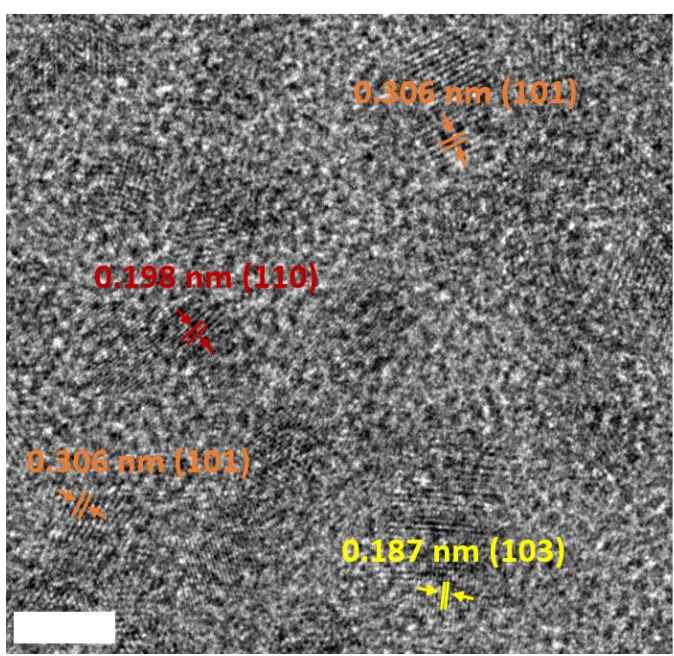

b

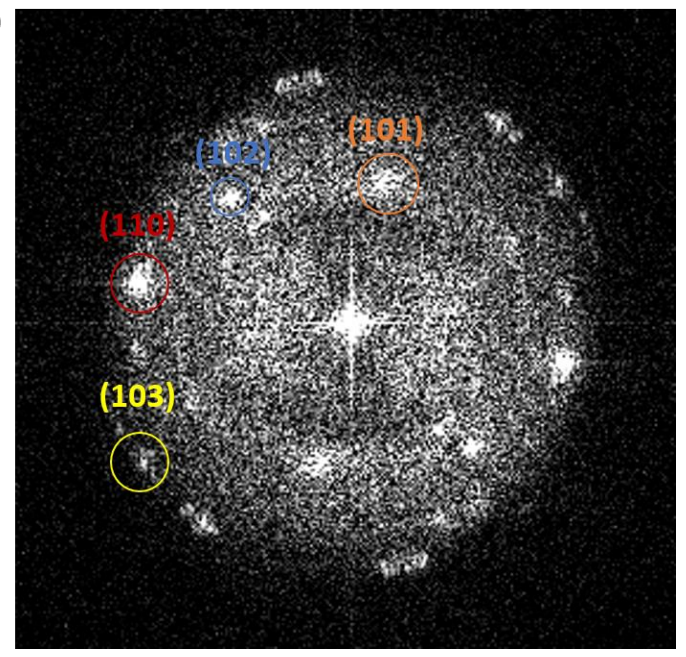

Figure S2. a) HRTEM image and b) corresponding FFT view of synthesized $L$-Cys-Cu $\mathrm{S}$ NPs. Scale bar $=5 \mathrm{~nm}$. 

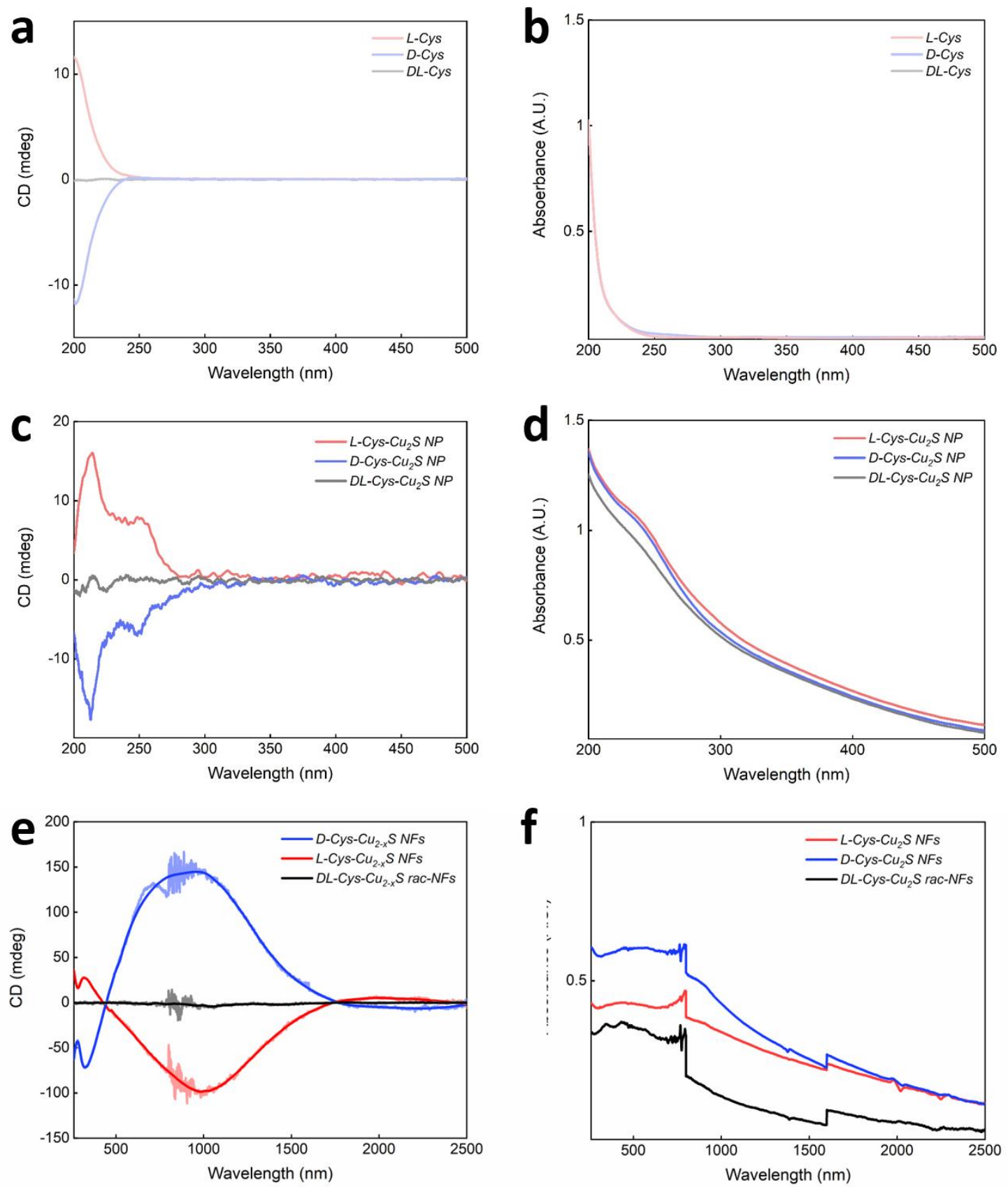

Figure S3. a-f) CD and UV-Vis-SWIR absorbance spectra of Cys (a, b), NPs (c, d), and NFs $(\mathrm{e}, \mathrm{f})$ (red for $L-$, blue for $D$-, and black for $D L-$ ). 

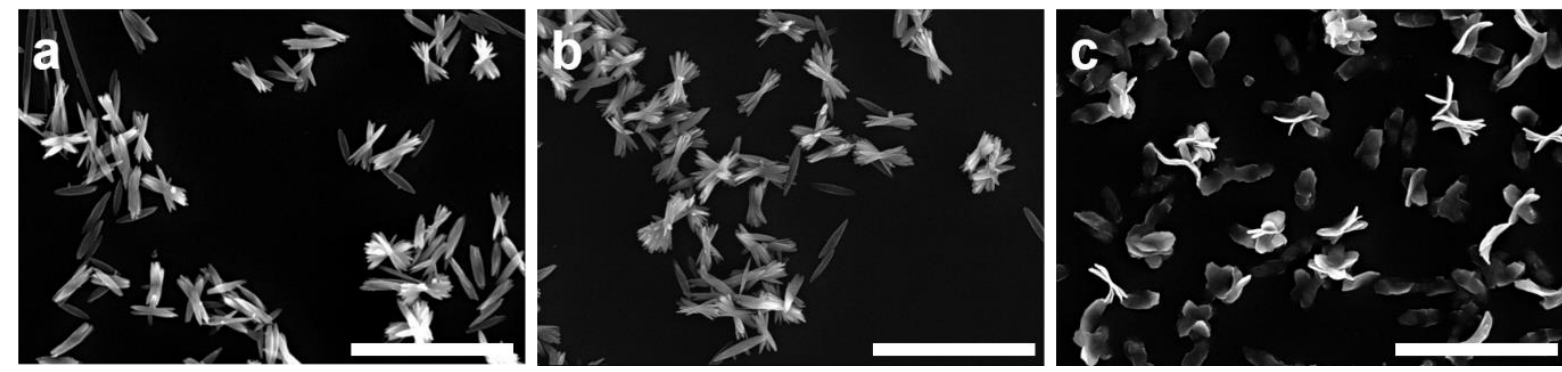

Figure S4. SEM images of a) $L-$, b) $D-C y s-\mathrm{Cu}_{2-\mathrm{x}} \mathrm{S}$ NFs and c) $D L-\mathrm{Cys}_{-}-\mathrm{Cu}_{2-\mathrm{x}} \mathrm{S}$ rac-NFs. Scale bars $=5 \mu \mathrm{m}$.
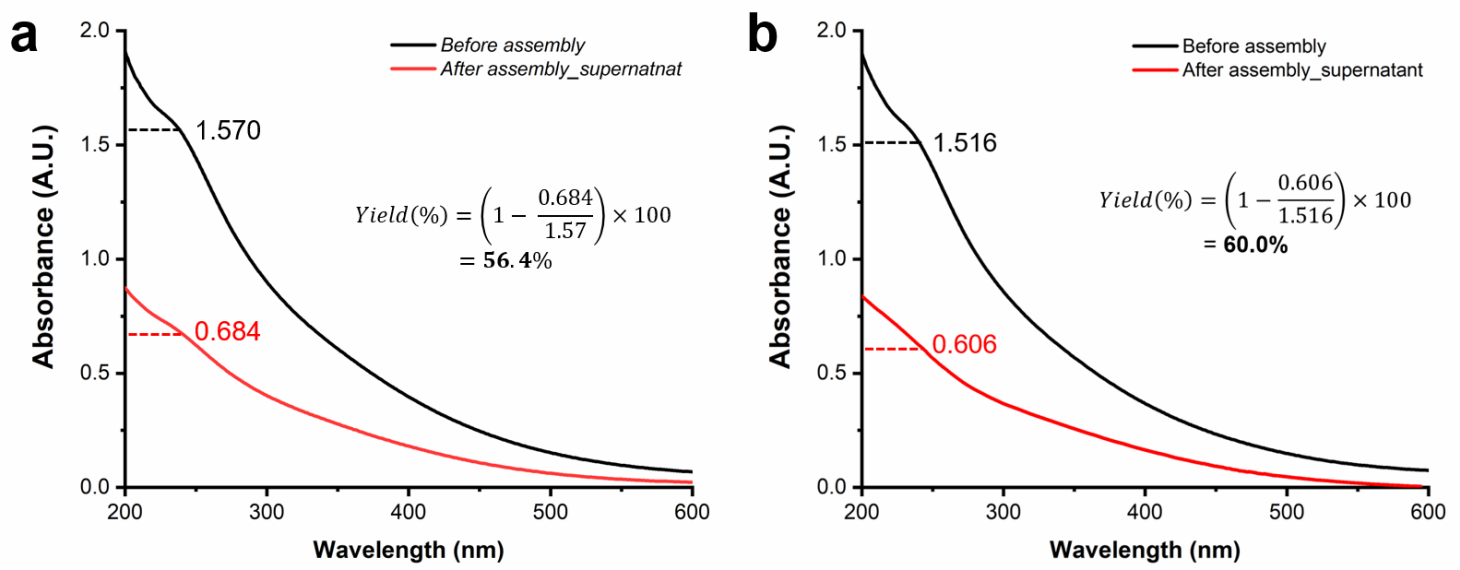

Figure S5. Calculated yield of a) $L-C y s-\mathrm{Cu}_{2-\mathrm{XS}} \mathrm{NFs}$ and b) $D$-Cys-Cu $\mathrm{Cu}_{2-\mathrm{x}} \mathrm{S}$ NFs. We calculated the yield based on the reference $1{ }^{1}$ As we can separate assembled NFs from unassembled NPs in solution by centrifugation after 20 hours aging process, we calculated the yield by comparing the concentration of initial and the final NPs from below equation. Due to the concentration is relevant to the intensity of UV-Vis absorbance spectra (Beer-Lambert laws, $A=\varepsilon b c, A$ : absorbance, $\varepsilon$ : molar extinction coefficient, b: beam path length, c: concentration), we used intensity of distinct peak of NPs at $249 \mathrm{~nm}$.

$$
\text { Yield }(\%)=\left(\frac{c_{0}-c}{c_{0}}\right) \times 100 \%=\left(1-\frac{c}{c_{0}}\right) \times 100 \%=\left(1-\frac{A}{A_{0}}\right) \times 100 \%
$$

( $c_{0}$ : Initial concentration of NPs before assembly, c: Final concentration of NPs after assembly, $A_{0}$ : Initial absorbance intensity of NPs at $249 \mathrm{~nm}$, A: Final absorbance intensity of NPs at $249 \mathrm{~nm}$ ) 

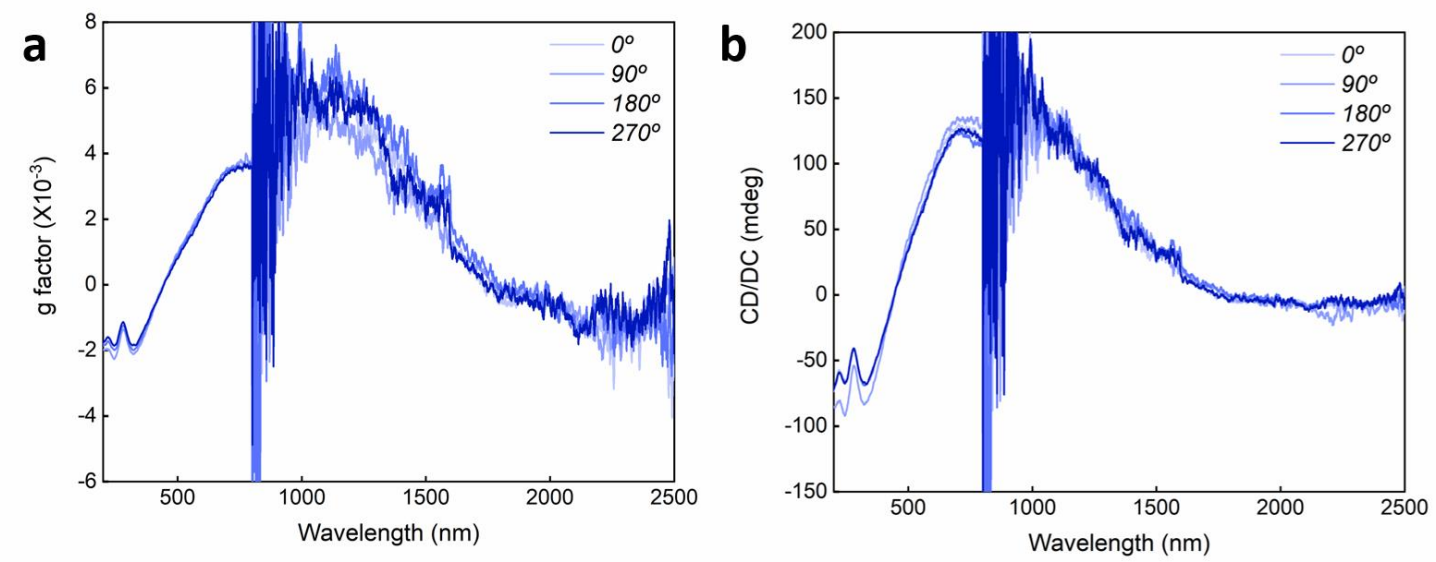

Figure S6. Angular dependence of CD spectra of $D$-Cys-Cu $-\mathrm{Cu}_{2-\mathrm{x}} \mathrm{S}$ NFs. a) $g$ factor and b) CD spectra measured by rotating the sample around its axes for $0^{\circ}, 90^{\circ}, 180^{\circ}, 270^{\circ}$.
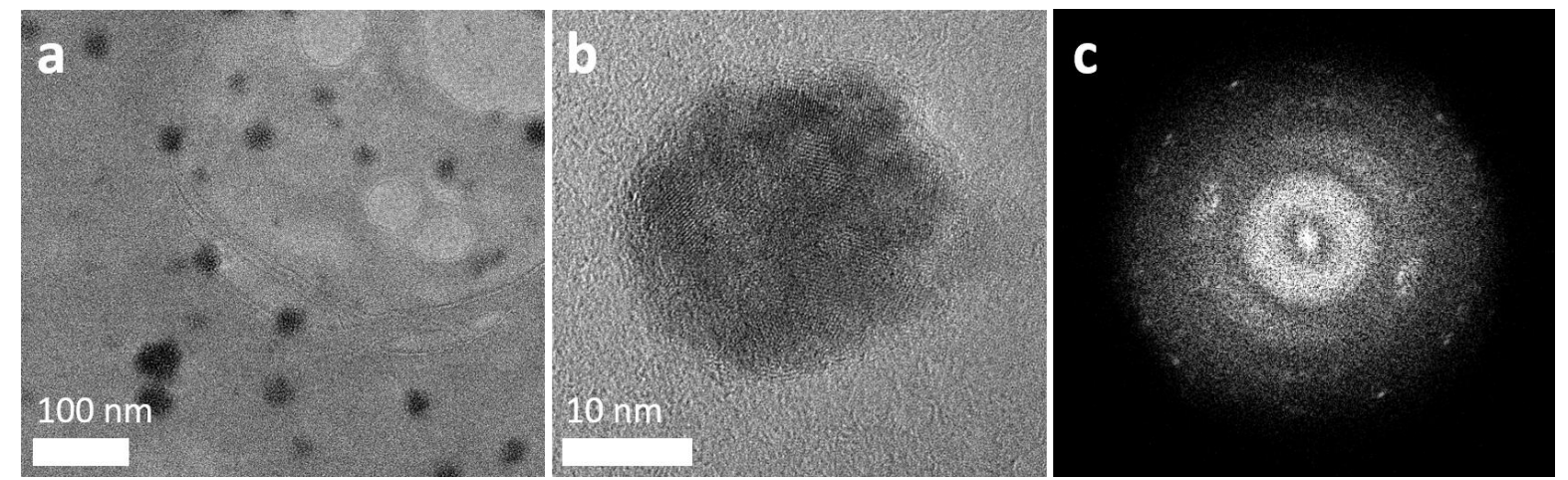

Figure S7. a) Representative TEM, b) HR-TEM image of single SP and c) corresponding FFT view.
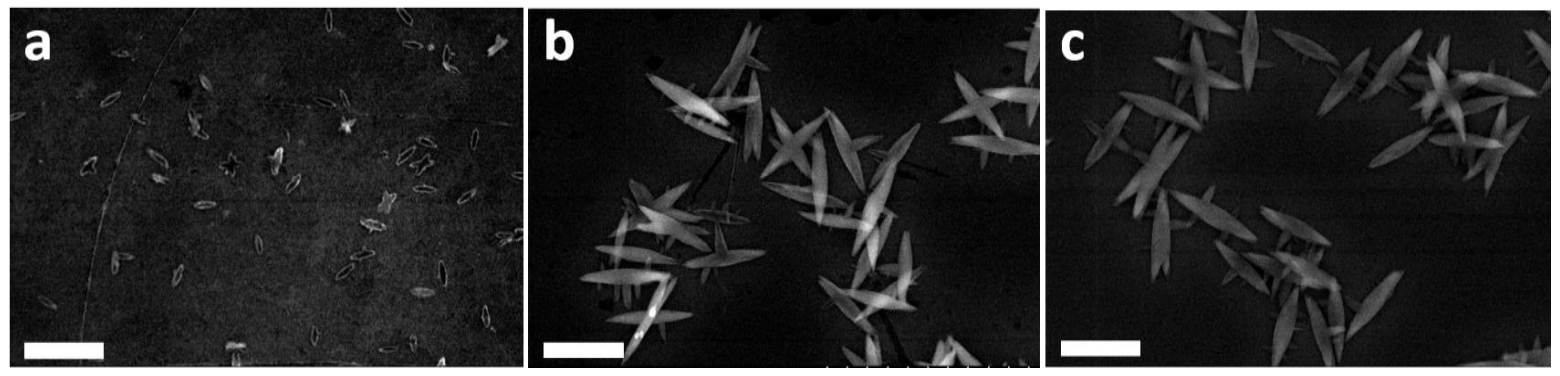

Figure S8. SEM images of NLs formed at different aging time. a) 2 hrs., b) 5 hrs., c) 10 hrs. Scale bars $=1 \mu \mathrm{m}$. 
a

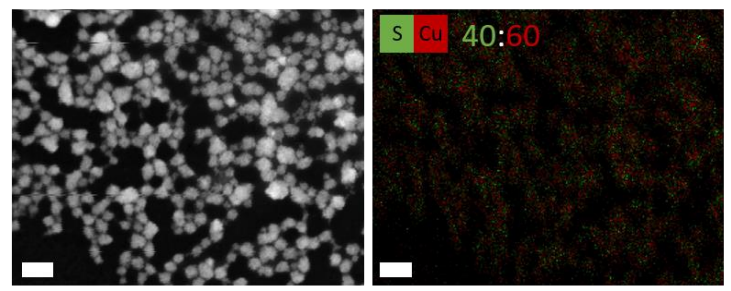

s

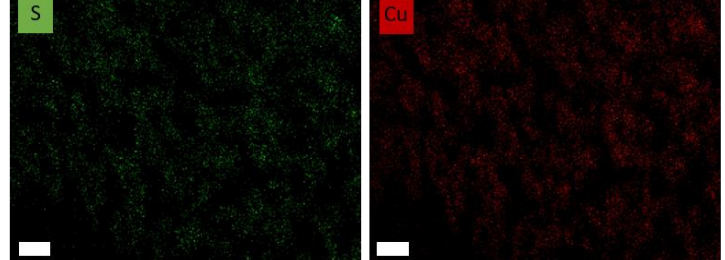

b
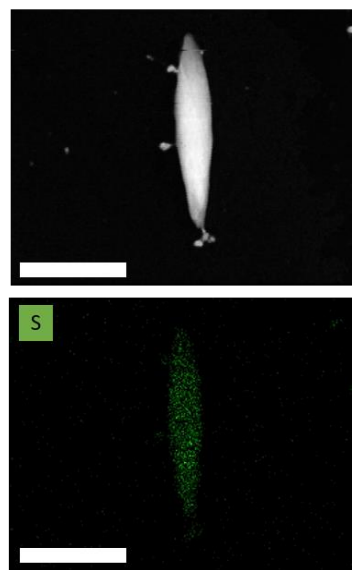
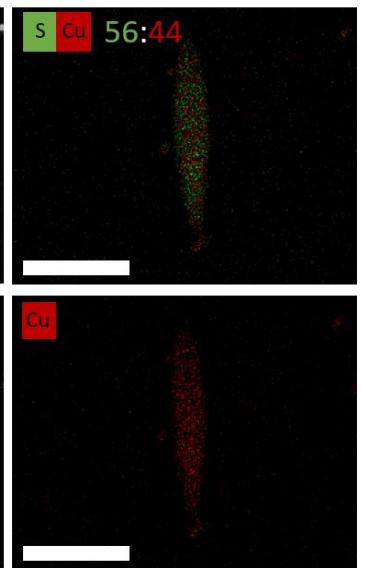

Figure S9. HADDF-STEM and EDS mapping images of a) supraparticles and b) nanoleaf. Scale bars $=100 \mathrm{~nm}$ and $500 \mathrm{~nm}$, respectively.
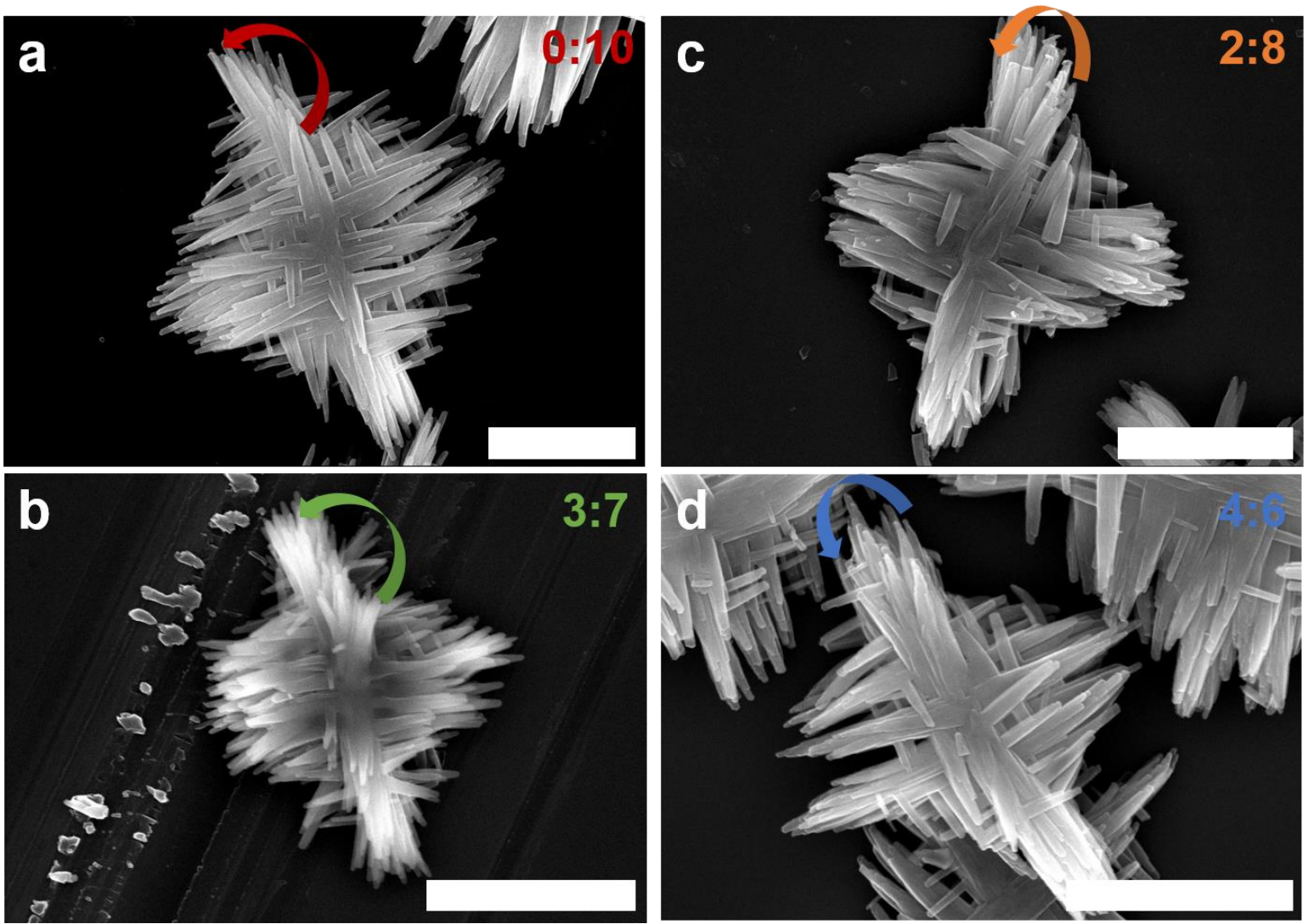

Figure S10. a-d) SEM images of nanostructures prepared with different TGA to $L$-Cys ratios: a) $0: 10$, b) $3: 7$, c) $2: 8$, d) 4:6, respectively (Scale bars $=2 \mu \mathrm{m}$ ). 

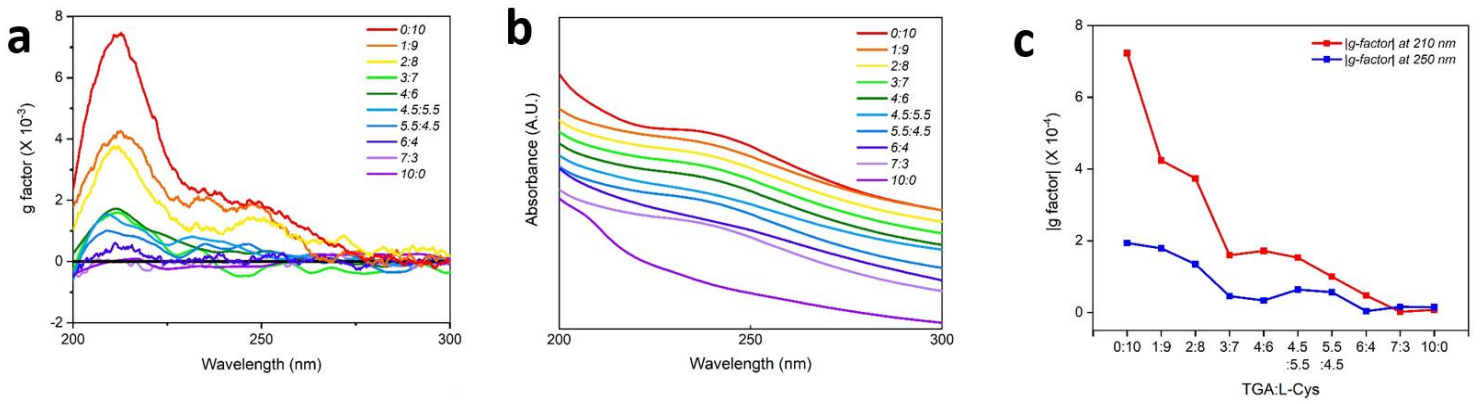

Figure S11. a) Optical activity ( $g$ factor) spectra and b) UV-Vis spectra of $L$-Cys-Cu $2 \mathrm{~S}$ NPs prepared with different TGA and $L$-Cys ratios. The corresponding spectra of absolute intensity of $g$ factor at $210 \mathrm{~nm}$ (red curve) and $250 \mathrm{~nm}$ (blue curve).
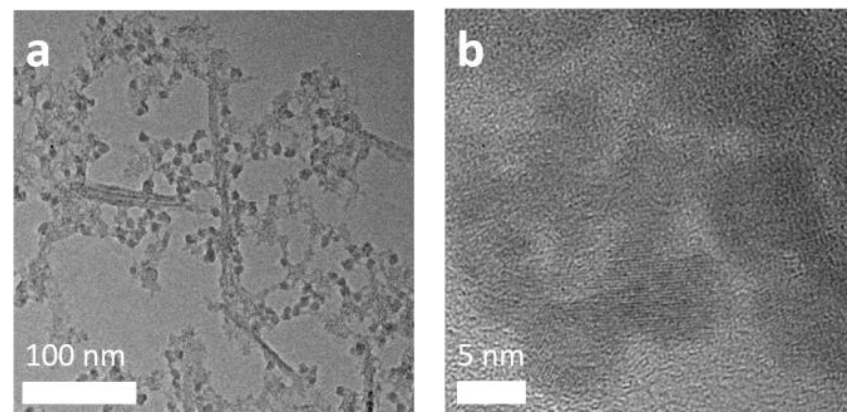

Figure S12. a) TEM and b) HR-TEM images of assembly of synthesized TGA-Cu $2 \mathrm{~S}$ NPs.

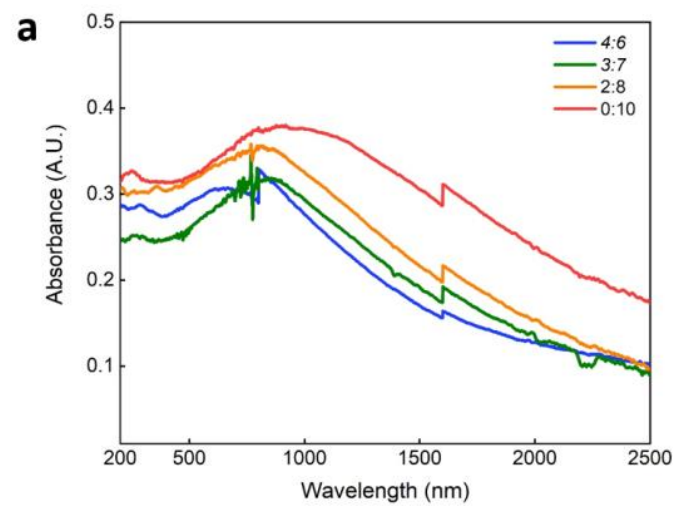

Figure S13. a) Absorbance spectra of assembled structures prepared with different TGA and $L$-Cys ratios. The spectral noises in the $800-850 \mathrm{~nm}$ and the $1600-1650 \mathrm{~nm}$ regions are created as the detectors change. 
a

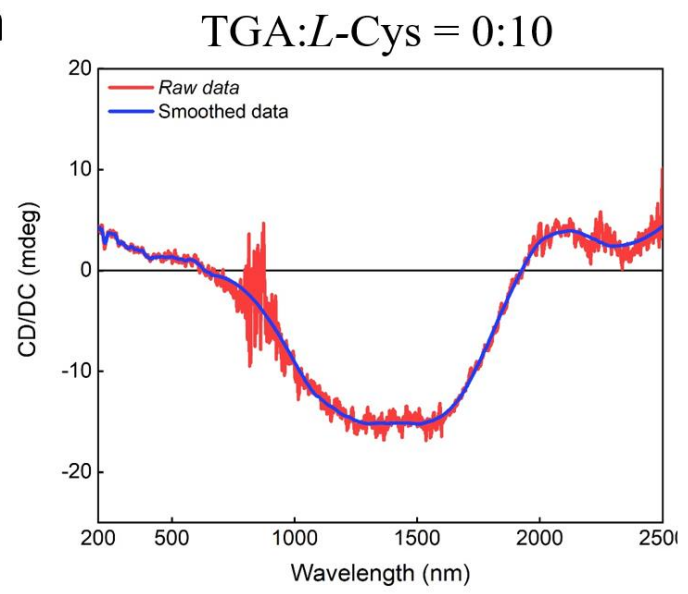

C

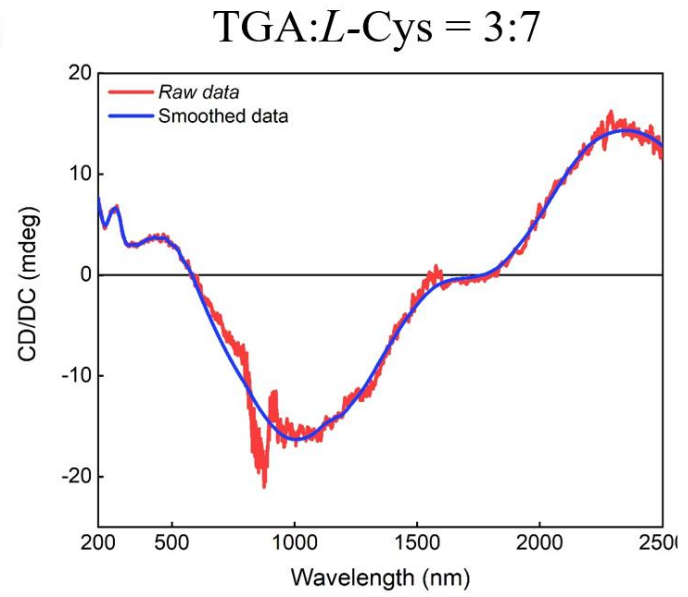

b

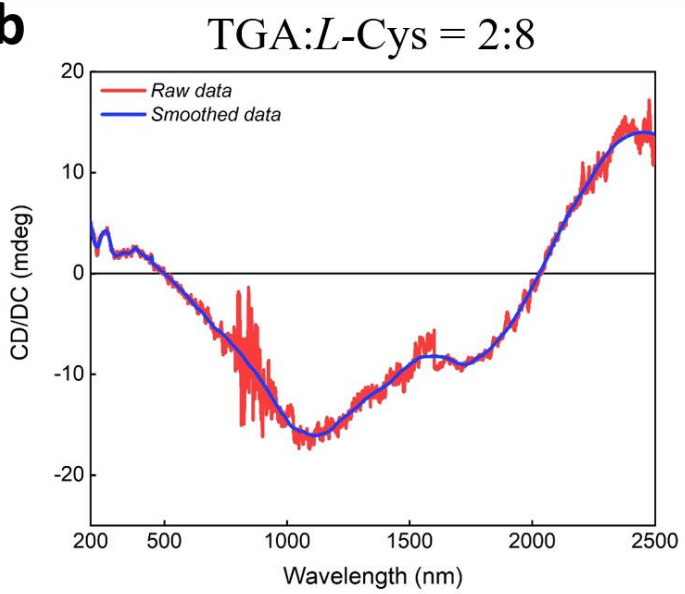

d $\quad$ TGA:L-Cys $=4: 6$

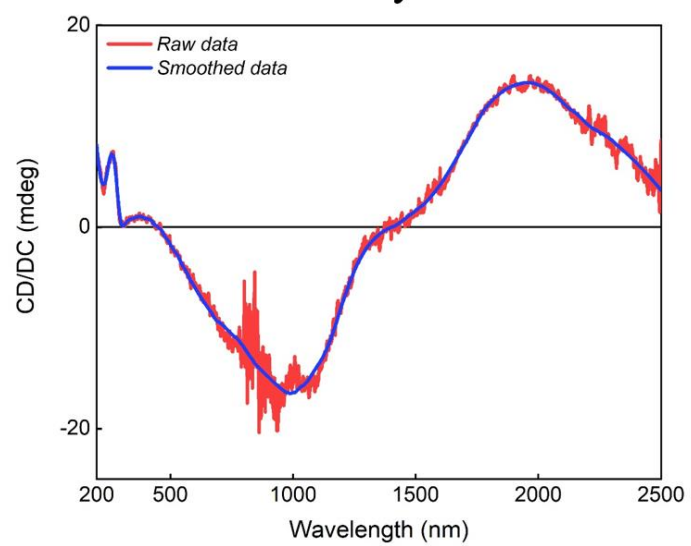

Figure S14. a-d) CD spectra of assembled structures prepared with different TGA and $L$-Cys ratios. TGA:L-Cys = a) $0: 10$, b) $2: 8$, c) $3: 7$, d) $4: 6$. The spectra were smoothed due to the detection transition noises at $800-850 \mathrm{~nm}, 1600-1650 \mathrm{~nm}$ region (raw data (red) smoothed data(blue)). 
a

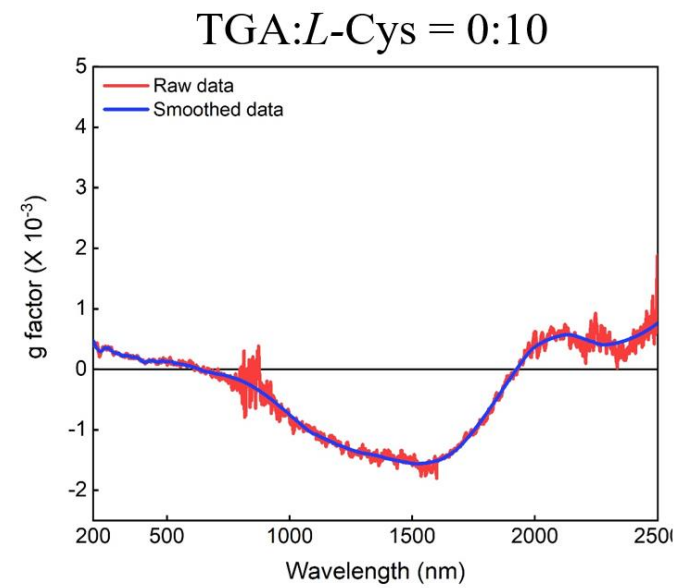

C

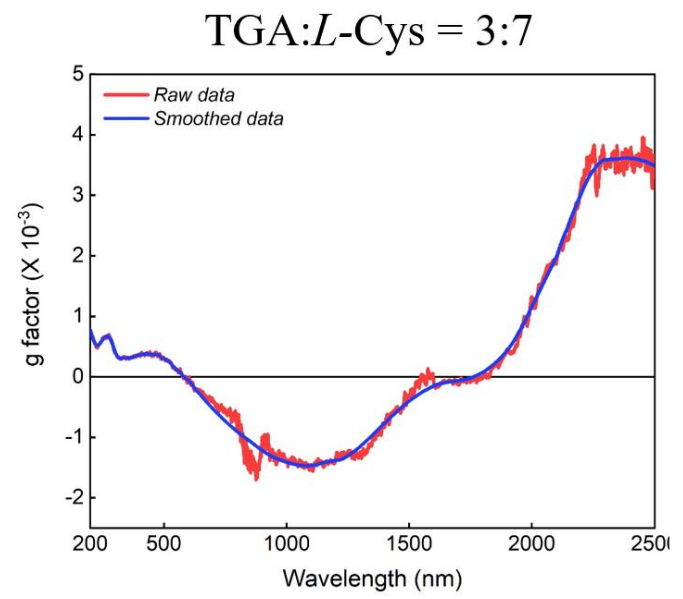

b

b TGA:L-Cys $=2: 8$

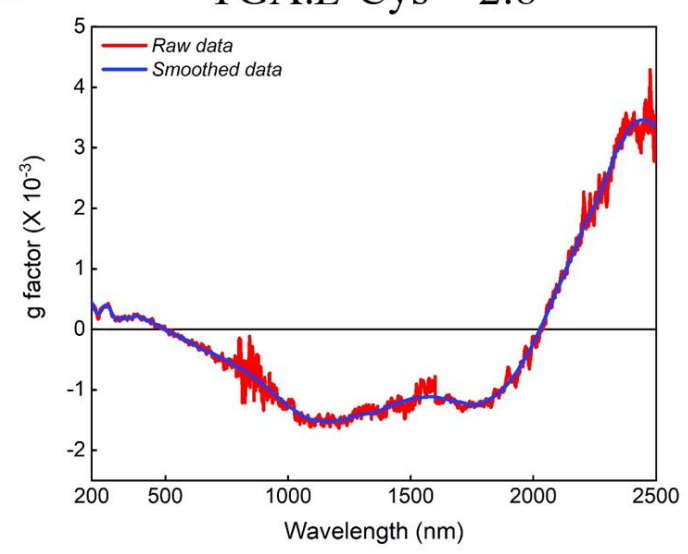

d

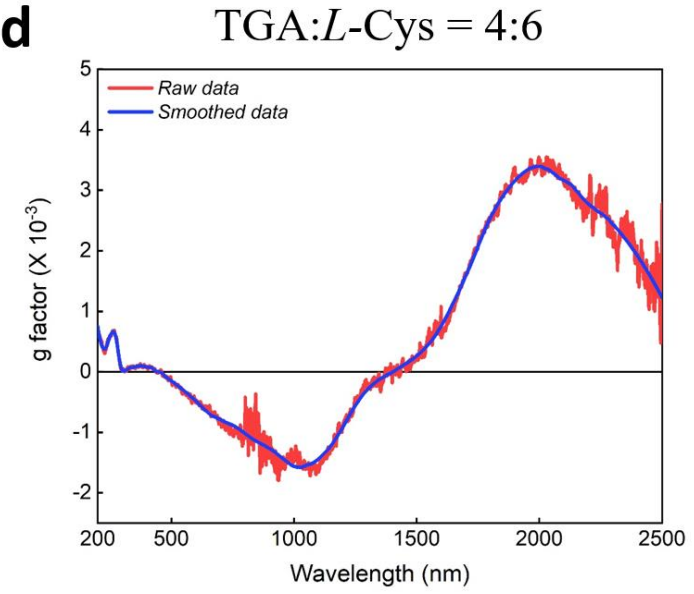

Figure S15. a-d) $g$ factor spectra of assembled structures prepared with different TGA and $L$ Cys ratios. TGA:L-Cys = a) 0:10, b) 2:8, c) 3:7, d) 4:6. The spectra were smoothed due to the detection transition noises at $800-850 \mathrm{~nm}, 1600-1650 \mathrm{~nm}$ region (raw data (red) smoothed data(blue)). 


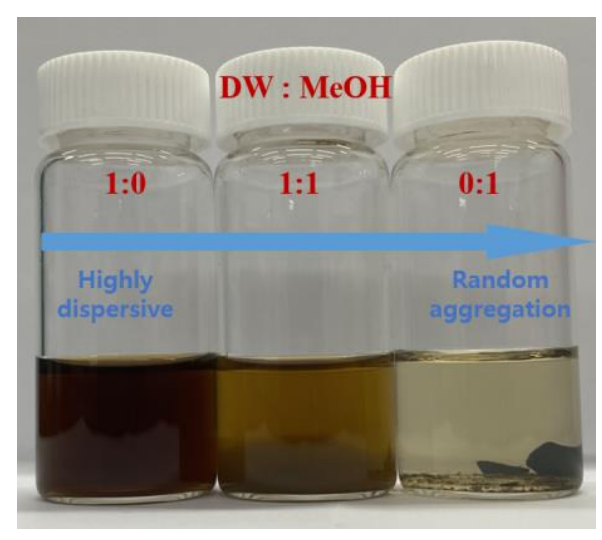

Figure S16. Self-assembly of NPs when the mixtures of DW and MeOH were used ( $\mathrm{V}_{\mathrm{DW}}$ : $\left.\mathrm{V}_{\mathrm{MeOH}}=1: 0,1: 1,0: 1\right)$. When $\mathrm{MeOH}$ was added, NPs randomly aggregated and their precipitants were observed.

References

(1) Yan, J.; Feng, W.; Kim, J. Y.; Lu, J.; Kumar, P.; Mu, Z.; Wu, X.; Mao, X.; Kotov, N. A. Self-Assembly of Chiral Nanoparticles into Semiconductor Helices with Tunable Near-Infrared Optical Activity. Chem. Mater. 2020, 32 (1), 476-488. 